\title{
Aristophanes venetus: i fratelli Rositini e la prima traduzione italiana del poeta comico greco (1545)
}

Aristophanes venetus : les frères Rositini et la première traduction italienne du poète comique grec (1545)

Aristophanes venetus: the Rositini brothers and the first italian translation of the greek comic poet (1545)

\section{Simone Beta}

\section{(2) OpenEdition}

\section{Journals}

Edizione digitale

URL: http://journals.openedition.org/cei/1346

DOI: $10.4000 /$ cei. 1346

ISSN: 2260-779X

\section{Editore}

UGA Éditions/Université Grenoble Alpes

\section{Edizione cartacea}

Data di pubblicazione: 1 novembre 2013

Paginazione: $57-70$

ISBN: 978-2-84310-234-9

ISSN: 1770-9571

Notizia bibliografica digitale

Simone Beta, "Aristophanes venetus: i fratelli Rositini e la prima traduzione italiana del poeta comico greco (1545)», Cahiers d'études italiennes [Online], 17 | 2013, online dal 01 mai 2015, consultato il 26 mars 2021. URL: http://journals.openedition.org/cei/1346 ; DOI: https://doi.org/10.4000/cei.1346 
ARISTOPHANES VENETUS:

\title{
I FRATELLI ROSITINI E LA PRIMA TRADUZIONE ITALIANA DEL POETA COMICO GRECO (1545)
}

\author{
Simone Beta \\ Université de Sienne
}

Oggi Pralboino è un sonnolento paese della Pianura Padana di quasi tremila abitanti non lontano dalla confluenza del piccolo fiume Mella col più grande Oglio, e di questo con l'ancora più grande Po, in un territorio che, dal punto di vista amministrativo, fa parte della provincia di Brescia e che si incunea in mezzo a due altre province che, come Brescia, appartengono alla Lombardia, vale a dire Cremona e Mantova. Il suo nome deriva, attraverso le forme "Prato di Alboino» e «Prat'Alboino», da quello del re longobardo Alboino che nel 569, dopo aver conquistato prima il Friuli e poi il Veneto, decise di assediare Brescia e pose il suo accampamento a circa trenta chilometri a sud-est della città. Donato da Desiderio, l'ultimo re longobardo, all'abate di Leno e da quest'ultimo, nei primi anni del 900, ad Acilao, il capostipite dei Gambara, il territorio (col nome di Pratalboino) rimase sempre legato al nome di questa famiglia - tanto che ancora oggi il suo monumento più celebre è proprio palazzo Gambara, benché le sue forme attuali settecentesche risalgano al rimaneggiamento voluto da Alemanno, che distrusse il castello di origine medievale nel quale era nata nella notte tra il 29 e il 30 novembre 1485 la figura più illustre della famiglia, la poetessa Veronica Gambara.

Figlia di Gianfrancesco e nipote dell'erudito Pietro, Veronica ricevette un'istruzione umanistica di buon livello che comprendeva sicuramente la conoscenza del latino e che, unita al suo talento poetico, le permisero di diventare una figura di spicco nel mondo letterario, apprezzata non solo dai suoi contemporanei (Pietro Bembo, Giangiorgio Trissino, Bernardo Tasso, Pietro l'Aretino e Ludovico Ariosto) ma anche dai posteri - tra i quali non si può non ricordare Giacomo Leopardi, che scelse un suo verso 
("Certi non d'altro mai che di morire») come esergo di una sua composizione giovanile, la cantica L'appressamento della morte ${ }^{1}$.

Veronica ebbe cinque fratelli: i quattro maschi furono Uberto (il primogenito, futuro cardinale), Ippolito, Brunoro (protonotario apostolico, legato ai pontefici Carlo V e Paolo III) e Camillo; l'unica sorella si chiamava Isotta. Di questi, Camillo condivise senźaltro con la sorella la passione per le letterature classiche, se è lui (come pare probabile) il Camillo Gambara al quale due medici di Pratalboino, Pietro e Bartolomeo Rositini, dedicarono la traduzione delle undici commedie di Aristofane, la prima traduzione integrale dell'opera omnia del comico greco in una lingua diversa dal latino.

Pubblicate a Venezia «apresso Vicenzo Vaugris, al segno d'Erasmo» nel 1545 col titolo Le comedie del facetissimo Aristofane, tradutte di Greco in lingua commune d'Italia, per Bartolomeo \& Pietro Rositini de Prat' Alboino, "con privilegio de lo Illustrissimo Senato Veneto per anni diece», sono dedicate "al nobile et virtuoso S. Camillo de Gambara» dai due traduttori (che si firmano «vostri sempre servitori Bartolomeo e Pietro Rositini de Prat' Alboino».

Nella lettera i due traduttori (che, di professione, facevano i medici) dicono al Gambara che, in segno di ringraziamento nei suoi confronti, hanno deciso di dedicare a lui la loro versione delle commedie di Aristofane - e non perché egli (che era un eccellente conoscitore della lingua greca) avesse bisogno di una traduzione italiana per comprendere le battute del poeta comico, ma perché si potesse semplicemente divertire senza alcuno sforzo $^{2}$.

Come si vede, manca in questa premessa un qualsiasi accenno al metodo seguito dai fratelli Rositini nel loro lavoro ermeneutico. Eppure Bartolomeo e Pietro partivano da presupposti molto chiari, che troviamo

I. Leopardi attribuisce erroneamente il verso a Vittoria Colonna (l'altra grande poetessa italiana del Cinquecento), ma esso proviene in realtà da una poesia di Veronica Gambara (la composizione 54, "Quando miro la terra, ornata e bella», v. 37), dalla quale lo stesso Leopardi ha preso in prestito un altro verso (v. I7: "Dico fra me pensando»,) per usarlo in una delle sue canzoni più famose, il Canto notturno di un pastore errante nell'Asia (v. 85).

2. «La grand'affettione e osservantia nostra verso voi Signor humanissimo, non hà mai sofferto, che de la vostra benignità e cortesia in niente s'habiam scordato. Onde noi per non parere dishumani ne smentichevoli di voi, che ne sete patrone, non sapendo qual cosa vi potesse esser più grata, poi ch'habiam fatto questa poca fatica in tradure in lingua propria e commune di tutta la Italia le Comedie di questo antico e faceto poeta, havemo deliberato mandarle fuori sotto il nome della S.V. Queste adunque publichiamo sotto il nome vostro, à ciò che, se così à voi parerà di fare, quando per gli studii sarete alquanto stanco, vi possiate con qualche suave $\mathrm{e}$ honesto spasso ristorare e alegrare, anchor che di queste Comedie in questa nostra lingua ridutte, in cosa niuna n'habiate bisogno per interpretation de 'l testo Greco, che siamo certissimi che senza traduttione alcuna e con facilità le Greche potete intendere: pur tutta via V.S. si degnarà d'accetar non quello dono ch'è un niente, ma l'animo nostro, che sempre è di farle ogni cosa che le sia à grado, in tutto ciò che noi possiamo fare.» 
esposti nella lettera «al lettore» che precede un'altra pubblicazione altrettanto impegnativa: Le vite degli illustri filosofi di Diogene Laertio, "da' 1 Greco Idiomate ridutte ne la lingua commune d'Italia», pubblicate in quello stesso anno presso il medesimo editore. L'opera è dedicata «al nobile et illustre Signore, il Signor Conte Giovan Francesco da Gambara», con parole che riecheggiano quelle usate per dedicare la traduzione di Aristofane a Camillo Gambara ${ }^{3}$.

Poiché Gianfrancesco è un nome ricorrente nella famiglia Gambara, non è facile capire con precisione quale sia il personaggio ricordato dai Rositini, perché il Gianfrancesco padre di Camillo (e della poetessa Veronica) era morto nel I5IO, mentre il Gianfrancesco figlio di Brunoro (e quindi nipote di Veronica Gambara), destinato a diventare un famoso cardinale, nel 1545 aveva appena compiuto dodici anni. Si tratta forse del discendente di un altro ramo della nobile famiglia bresciana, il conte che sappiamo aver accolto nel 1522 in casa sua il filosofo Mario Nizzòli (più noto col nome latinizzato Nizolius), nato nel I488 o nel I498 a Brescello, futuro professore nell'università di Parma, che in segno di gratitudine gli avrebbe dedicato la sua opera più famosa, le Observationes in M. T. Ciceronem, stampate nel 1535 proprio a Pralboino a spese degli editori Matteo e Camillo Avogadro 4 .

Ma quel che ci importa sono, come dicevamo, le parole che i fratelli Rositini rivolgono ai lettori per spiegare gli obiettivi che hanno cercato di perseguire con la loro traduzione:

Ogni mediocre ingegno può molto ben conoscere che difficultà sia l'interpretar d'una in un'altra lingua, et quanto più tra se sono lontane, tanto in quelle nasce la difficultà magiore. Onde se noi scrivendo, come hogidì s'usa in Italia, haveremo usato parole

3. «Havendo noi ne i passati tempi che siamo stati in Venetia, per nostra essercitatione tradutte le vite d' i Filosofi di Diogene Laertio Grece, in lingua commune d'Italia, con quella diligenza magiore, che à noi è stato possibile, e fattole stampar à commun'utilità de chi non sa lettere ne Grece, ne Latine: ne è parso conveniente cosa, mandarle in luce sotto il nome non d'altra persona che de la S.V. di certo non perché quella habia bisogno di tal nostra tradottione, per essere dottissima ne l'una e l'altra lingua, e scientissima de le cose di filosofia, ma per essere nostro patrone e signore singolare: à cui siamo obligati, non solamente tutte le nostre fatiche, ma la vita propria anchora. La onde le dedichiamo la stessa tradottione, supplicandole ad accettarla benignamente, e non riguarda tanto à la qualità de 'l dono, quanto à l'animo e affettion nostra ver la S.V. à cui baciandole le mani humilissimamente si raccommandiamo.»

4. Su questo Gianfrancesco Gambara cfr. G. Tiraboschi, Biblioteca modenese o Notizie della vita e delle opere degli scrittori natii degli stati del serenissimo signor Duca di Modena, tomo 3, Modena, Società tipografica, I783, p. 350. Sul Nizzoli e sulla sua opera ciceroniana (il primo lessico latino costruito interamente sulla base delle opere dell'autore latino, costituito da oltre ventimila lemmi), cfr. la voce del Dizionario biografico degli italiani redatta da Margherita Palumbo (vol. 78, pp. 620-623); cfr. anche i cinque articoli pubblicati da G. Pagani in Rendiconti della Reale Accademia dei Lincei, s. 5, II, I893 ("Mario Nizzoli e il suo lessico", pp. 554-575; "Le polemiche letterarie di Mario Nizzoli», pp. 630-660; "Mario Nizzoli umanista e filosofo del sec. XVI», pp. 716-74I; "Operosità letteraria di Mario Nizzoli», pp. 819-826; "Gli ultimi anni di vita di Mario Nizzoli», pp. 897-922). 
che sono in uso, de le quali la magior parte i scrittori de la lingua Italiana fin'hora non hanno usato, niuno punto si devrà meravigliare. Per ciò che come interpreti et traduttor fideli, non havemo voluto epitomar, né parafrasticare: anzi, in quanto sia mai stato possibile, ne 'l tradur, sempre habiam servato quella fedeltà che si conviene a i veri e fideli interpreti. E per che il proposito nostro sempre è stato di farsi intendere ne le nostre scritture: non parole più d'un paese che de l'altro de la Italia havemo usato, ma in somma quelle che a tutta la Italia universalmente sono proprie et communi. Si che lettori humanissimi, se in queste nostre traduttioni vi sarà cosa che a pieno non ne restate sodisfatti (ben che quelle, ov'era la difficultà magiore, con l'aiuto d'alcune persone e dotte le havemo fatte facili e intelligibili) co l' vostro benigno animo potrete far conferire il testo volgar co 'l Greco, donde è stato cavato. E così restarete su quello sodisfatti.

L'importanza di queste riflessioni è stata ben colta da Riccardo Tesi che vi ha visto «un modo, prettamente strumentale, di tradurre i classici greci, che volentieri rinuncia agli abbellimenti stilistici e preferisce invece concentrarsi sulla restituzione precisa, quasi parola per parola (come il didattico transferre ad verbum degli umanisti quattrocenteschi), del testo antico», che si poggia su due punti fondamentali: «l'uso di forme straniere (grecismi compresi) non autorizzate da una consolidata tradizione letteraria, ma tuttavia coerenti negli impieghi non letterari (la lingua degli 'scrittori' è contrapposta a quella scritta che 'hogidì s'usa')» e «l'adozione di un criterio di selezione lessicale che rinunci a parole di diffusione locale o regionale»'s.

Tuttavia, tradurre un prosatore non è la stessa cosa che tradurre un poeta - ed è per questo che nel portare le commedie di Aristofane dal greco all'italiano i fratelli Rositini non si sono attenuti ai medesimi principi che hanno seguito nel portare dalla lingua antica alla moderna le biografie di Diogene Laerzio; se per la prosa hanno rinunciato ai termini dialettali per dare la preferenza a quelli «nazionali» (anche se una nazione italiana ancora non c'era, e non ci sarebbe stata per altri tre secoli), per la poesia non l'hanno fatto, così come non l'aveva fatto lo stesso Aristofane, che spesso aveva utilizzato per scopi comici, accanto al consueto dialetto attico, anche espressioni dialettali provenienti da altre aree linguistiche sia greche (parole in dialetto laconico, megarese e beotico) sia «barbare» (parole vicine alla lingua parlata dai Persiani e dagli Sciti) ${ }^{6}$.

5. R. Tesi, Aristotele in italiano. I grecismi nelle traduzioni rinascimentali della 'Poetica', Firenze, Accademia della Crusca, I997, p. 2I.

6. Sull'uso del dialetto in Aristofane cfr. S. Colvin, Dialect in Aristophanes. The Politics of Language in Ancient Greek Literature, Oxford, Clarendon Press, 1999. 
In questo articolo passerò rapidamente in rassegna alcuni aspetti della traduzione di una delle più famose commedie di Aristofane, le Nuvole, rappresentate nel 423 , mettendola a confronto con un'altra versione ben più famosa, perché si tratta della prima traduzione in lingua francese, che si deve ad Anne Le Fèvre, più nota col nome di Madame Dacier, figlia di un eccellente classicista, il filologo Tanneguy Le Fèvre, che amava farsi chiamare Tanaquillus Faber e che aveva a sua volta tradotto (in latino, però!) una commedia dello stesso Aristofane ${ }^{7}$.

Cominciamo dal prologo della commedia, che vede il protagonista, il vecchio Strepsiade, lamentarsi della passione smisurata del figlio Fidippide per i cavalli, che lo porta a fare in continuazione una serie di debiti (regolarmente pagati dal padre). Ecco il testo dei primi ventiquattro versi nella traduzione francese di quella che è stata per anni l'edizione standard del testo di Aristofane ${ }^{8}$ :

Hélas! Hélas! Ô Zeus souverain, quelle longueur ont ces nuits! Cela n'en finit pas! Il ne fera donc jamais jour? Et pourtant, il y a longtemps que j'ai entendu le coq. Et mes esclaves ronflent! Il n'en ềt pas été ainsi autrefois. Maudite sois-tu, ô Guerre, pour mille raisons! Il ne m'est même pas permis de châtier mes esclaves. Lui non plus, l'honnête jeune homme que voilà, ne se réveille de la nuit, mais il pète, dans cinq fourrures entortillé en boule (Il se recouche.) Allons, si vous permettez, ronflons bien enveloppé. Mais, malheureux, je ne puis dormir, rongé que je suis par... la dépense, la mangeoire et les dettes, à cause de ce fils-là! Lui, portant longue chevelure, monte à cheval, conduit un attelage, rêve chevaux; moi, je dépéris en voyant la lune ramener les jours de vingtaine; car les intérêts courent. (Il réveille un de ses esclaves.) Petit, allume la lampe; va me tirer mon livre de comptes, que jy lise tous ceux à qui je dois et fasse le calcul des intérêts. (L'esclave obéit.) Ah ça, voyons, qu'est-ce que je dois? Douze mines à Pasias. Pourquoi douze mines à Pasias? Pourquoi ai-je emprunté?.. C'est quand j'achetai le cheval au Coppa. Ah misère! Que n'ai-je plutôt "écopé" un coup de pierre à l'oil!

7. La traduzione di Mme Dacier (Le Plutus et les Nuées d'Aristophane, comédies grecques) fu pubblicata a Parigi dagli editori Denys Thierry e Claude Barbin nel I684; dieci anni prima il padre aveva pubblicato a Saumur ("typis \& sumptibus Isaaci Desbordes \& Ioannis Lesnerii») insieme alle sue lettere la traduzione latina delle Ecclesiazuse (Tanaquilli Fabri Epistolae, Pars posterior, Editio altera priori emendatior. Additae sunt Aristophanis EKK $\Lambda \mathrm{H} \Sigma \mathrm{IAZOY} \Sigma \mathrm{AI}$ cum interpretatione nova, notis \& emendationibus). Nel I683 Mme Dacier (che nel corso della sua vita tradusse anche altri autori greci del calibro di Omero, Saffo, Anacreonte, Plauto e Terenzio) aveva sposato il migliore allievo di suo padre, André Dacier, anch'egli valente traduttore (famosa la sua versione francese delle Vite parallele di Plutarco). Su Mme Dacier cfr. i seguenti saggi: P. Mazon, Mme Dacier et les traductions d'Homère en France, Oxford, Clarendon Press, 1936; E. Malcovati, Madame Dacier: una gentildonna filologa del gran secolo, Firenze, Sansoni, 1952; F. Farnham, Madame Dacier: Scholar and Humanist, Monterey, Angel Press, 1976; G. S. Santangelo, Madame Dacier, una filologa nella crisi: 1672I720, Roma, Bulzoni, I984.

8. Il testo greco di Victor Coulon e la traduzione francese di Hilaire Van Daele sono stati pubblicati a Parigi nella Collection Budé (Aristophane, Comédies, Tome I: Les Acharniens, Les Cavaliers, Les Nuées) nel 1923, pubblicati una seconda volta con alcune correzioni nel $1934 \mathrm{e}$ da allora più volte ristampati. 


\section{La traduzione di Mme Dacier è la seguente:}

Hai, hai, mon Dieu, que le nuits son longues! Le jour ne paroîtra-t'il donc jamais? Il $y$ a déja long-temps que j'ay oüy le chant du Coq, \& mes Valets ronflent encore comme sil n'estoit que minuit! Ils n'en usoient pas ainsi autre fois, que maudit soit la guerre, pour mille raisons, mais principalement parce qu'il ne m'est pas permis de châtier ces coquins! Et ce brave fils que j'ay là séveille-t'il plus que les autres? Ne ronfle-t'il pas aussi empaqueté dans une douzaine de couvertures? Mais voyons un peu si en nous enfonçant aussi dans le lit, nous ne pourrions point ronfler comme eux... helas il n'y a pas moyen, je me sens déchirer de tous costés. La dépense que je suis obligé de faire, tous ces chevaux qu'il me faut nourrir, tout ce que je dois à cause de ce beau fils, ne me permet pas de fermer l'ail. Et ce n'est pas ce qui le met en peine, il ne pense qu'à ajuster les cheveux, \& à faire des courses de chevaux \& de chariots, c'est-là ce qui luy tient au coeur. Toute la nuit il ne réve qu'à ses chevaux \& à sa chevalerie, \& moy je meurs de chagrin, car voicy le jour qu'il faut payer les interests. Hola, Dromon, allume ma lampe \& me donne mon livre, afin que je compte \& que je voye à qui je dois. Douze mines à Pasias. Douze mines à Pasias! D’où dois-je ces douze mines? Pourquoy les ay-je empruntées? Ho, je m'en souviens, ce fut quant j'achetay ce cheval. Ah, que je suis malheureux! Que ne me crevay-je plûtot un oil!!

\section{E come vengono tradotti questi versi dai fratelli Rositini nell'incipit della commedia da loro intitolata Le nebule? Così:}

Oimè, oimè, o signor Giove quanto sono lunghe le notti: non si farà hormai dì! E pur un buon pezzo è, ch'io ho udito il gallo, et i famigli runchegiano, ma non già da qui in dietro. $\mathrm{O}$ guerra rea per causa de molti, vatene in mal'hora, per ciò che a me non è lecito a punire i servidori. Ma ne anche questo da ben giovane di notte si lieva, anzi poltronegia in cinque schiavine involtosi. E se a noi pare, runchegiamo coperti: ma io infelice già dormir non posso, punto, e morduto da la spesa, e da la stalla, e da 'l debito, per questo mio figliuolo. Et egli con suoi bei capelli se ne cavalca, e su 'l cocchio si fa menare, e cavalli s'insogna: io poi mi muoro, vedendo che la Luna s'invecchia: però che le usure s'approssimano. Impizza regazo il lume, e portami il libro da i conti, che voglio sapere a quanti sono debitore, et voglio vedere il conto de le usure. Sù, ch'io vega quel che son debitore. Dodeci mine a Pasia: in che modo dodeci mine a Pasia? Ch' ho io adoperato? Quando comprai io cavallo bollato co '1 $\mathrm{k}$ ? Ahime sventurato, piacesse $\mathrm{a}$ i dei che m'havesse tratto fuora più presto un'occhio con questo sasso. ${ }^{\text {Io }}$

9. All'altezza della parte finale della battuta, nel margine sinistro la Dacier aggiunge (come fa di solito nella sua edizione) la seguente didascalia: "Il dit cela en songeant en luy-mesme \& avant que d'avoir son livre."

Io. Diversamente dall'edizione di Mme Dacier ( $\mathrm{e}$ anche, com'è naturale, da quella di Coulon), nella traduzione dei fratelli Rositini non ci sono le didascalie e non c’è nessuna divisione né in atti né in scene (il testo scorre continuo, con la sola indicazione del personaggio che pronuncia la battuta); l'elenco dei personaggi viene riportato prima del prologo, subito dopo il titolo (Strepsiade, Fidippide, Servidor di Strepsiade, Discepolo di Socrate, Socrate, Coro de le Nebule, Parola giusta, Parola ingiusta, Creditore Pasia, Il testimonio, Un'altro creditore Aminia, Cherefonte), mentre in Mme Dacier i personaggi non solo sono elencati all'inizio, con l'aggiunta di una breve descrizione (Strepsiade è definito "villageois, père de Phidippide»), ma vengono sempre indicati con precisione prima di ogni scena e corredati di un nome proprio, anche quando nell'originale greco

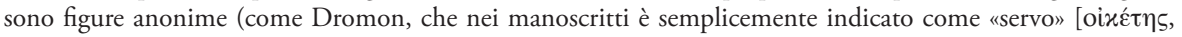


Se si confrontano le due traduzioni francesi, si nota subito come Mme Dacier non miri a rendere il testo in modo letterale, ma si prenda di tanto in tanto qualche piccola libertà per rendere la sua versione più scorrevole e non dover ricorrere alle note esplicative che, spesso, appesantiscono le traduzioni dei classici (soprattutto le traduzioni delle commedie di Aristofane, che fanno continui riferimenti più o meno allusivi a situazioni contemporanee a noi poco comprensibili, o che amano giocare con le parole) ${ }^{\text {II: }}$ : per questo, negli ultimi versi, Mme Dacier sorvola sul

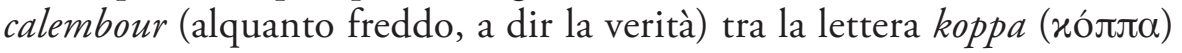
che contraddistingueva il cavallo di razza comprato da Fidippide e il verbo kopto $($ xó $\tau \tau \omega)$ che significa "colpire».

Fedeli nella loro adesione al testo, i fratelli Rositini scelgono invece di tradurre tutto, col risultato di pubblicare un testo non molto comprensibile che non fa nemmeno intuire il gioco di parole di Aristofane. In altri casi, Mme Dacier si limita a semplificare il testo: l'esclamazione iniziale, che in italiano è resa più o meno correttamente con "o signor Giove» (e che Van Daele traduce con estrema precisione "ÔO Zeus souverain»), diventa semplicemente "mon dieu»; poco più avanti, il termine neutro «servi» («servidori» nell'italiano — con sfumature venete — dei Rositini) assume una coloratura plautina («coquins»); ancora, le «cinque coperte» del testo greco diventano «une douzaine».

Se invece esaminiamo più da vicino la traduzione italiana, vi troviamo alcune interessanti particolarità che caratterizzano tutto il lavoro dei fratelli Rositini, non solo in questa ma anche nelle restanti commedie. Una di queste caratteristiche è l'utilizzo anacronistico di vocaboli che rimandano più ai tempi e ai luoghi dei due traduttori che non all'Atene del $\mathrm{v}$ secolo a.C. Per indicare le coperte appena menzionate nelle quali si avvolge Fidippide per continuare il suo sonno, non viene usato il semplice "coperta» (come fa Mme Dacier, che traduce con «couverture»), e nemmeno un termine che si avvicini al significato preciso del termine greco ( бıбú $\alpha$, sisyra), che indicava una sorta di pelliccia fatta con il vello di una capra (come fa Van Daele, che traduce con «fourrure»): le "coperte» sono chiamate «schiavine», un termine che indicava, tra le altre cose, proprio le coperte da letto fatte di tessuto grossolano ${ }^{12}$.

oiketes], o come più avanti Cenagoras, il discepolo di Socrate che introduce Strepsiade nel "pensatoio», che è solo un «allievo» [ $[\mu \alpha \theta \eta \tau \dot{\zeta}$, mathetes]).

II. Nell'edizione di Coulon, le note esplicative sono frequenti: solo in questi ventiquattro versi ce ne sono ben cinque!

I2. Cfr. il secondo significato riportato nel Grande dizionario della lingua italiana fondato da Salvatore Battaglia (Torino, UTET, 196I-2002, vol. XVII, s.v.), testimoniato in autori come San Bernardino da Siena, 
Sempre in questo passo possiamo sottolineare come la resa del parti-

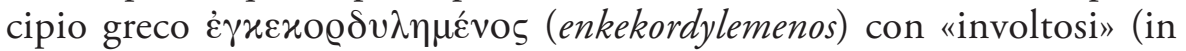
italiano meno arcaico, «avvolto») non renda tutte le sfumature dell'originale, alle quali si avvicinano di più sia il participio "empaqueté» usato da Mme Dacier sia, soprattutto, la perifrasi «entortillé en boule» di Van Daele, perché il verbo (una delle innumerevoli invenzioni di Aristofane) è costruito

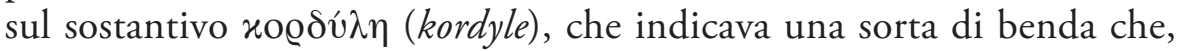
quando veniva usata come un turbante o una fasciatura, finiva per assomigliare a un fagotto. La banalizzazione dei Rositini ha un antecedente (probabilmente diretto): nella prima traduzione integrale latina del teatro di Aristofane pubblicata nel 1538 dal misterioso erudito di Capodistria Andreas Divus, il participio è reso proprio con involutus; poiché in molti punti la traduzione dei Rositini sembra perfettamente modellata su quella del Divus, è lecito supporre che anche il loro involtosi derivi da là ${ }^{13}$.

Se qui i Rositini, nel seguire il Divus, si limitano a peccare di imprecisione, altrove commettono invece veri e propri errori, che costituiscono purtroppo il limite più grande della loro versione, spesso incomprensibile e sgrammaticata. La loro enigmatica traduzione «ma non già da qui in dietro" per il corretto "Il n'en ềt pas été ainsi autrefois» (Van Daele) si deve tanto alla loro non adeguata conoscenza del greco quanto all'influsso dell'altrettanto enigmatico "sed non ante hac» del Divus; lo stesso vale per il loro «O guerra rea per causa de molti» (Van Daele: «Maudite sois-tu, $\hat{o}$ Guerre, pour mille raisons!»), molto probabilmente influenzato dal testo ambiguo stampato dal Divus ("Pereas scilicet o bellum multorum gratia»), che, com'era sua consuetudine (e com'era consuetudine degli umanisti in generale), traduceva verbum de verbo. In un altro caso, però, l'errore è tutto dei fratelli Rositini: non avendo compreso che l'espressione greca «en voyant la lune ramener les jours de vingtaine» (Van Daele) significava che era giunto il momento di pagare gli interessi ai debitori (come invece

Vespasiano da Bisticci, Leone Africano e Giovanbattista Marino; il significato più comune di schiavina era «mantello di tessuto grossolano e fornito di maniche e cappuccio usato specialmente nel Medioevo da viaggiatori, pellegrini ecc. per ripararsi dalle intemperie e dal freddo».

13. Sulla traduzione di Andreas Divus (Aristophanis, comicorum principis, Comoediae undecim, è Graeco in Latinum, ad verbum translatae, Andrea Divo Iustinopolitano interprete, Venetijs: apud d. Iacob a Burgofrancho Papiensem, I538, più ristampata da vari editori per tutto il secolo), cfr. S. Beta, «La prima traduzione latina della Lisistrata. Luci e ombre della versione di Andrea Divo", Quaderni Urbinati di Cultura Classica, n.s. Ioo, n. I, 2012 (vol. I29 della serie continua), pp. 95-II4. Un preciso confronto tra la traduzione delle Nuvole del Divus e quella dell'umanista Nicodemus Frischlin (Nicodemi Frischlini Aristophanes, veteris comoediae princeps: poeta longe facetissimus et eloquentissimus: repurgatus a mendis, et imitatione Plauti atque Terentii interpretatus, ita ut fere Carmen Carmini, numerus numero, pes pedi, modus modo, Latinismus Graecismo respondeat (Frankfurtam-Main, Johannes Spies, 1586) si potrà leggere alla fine del 2013 in J. Nassichuk, 'Strepsiades' Latin Voice: Two Renaissance Translations of Aristophanes' Clouds, in corso di stampa. 
comprenderà bene Mme Dacier scegliendo di parafrasare l'espressione «le jour qu'il faut payer les interests»), hanno tradotto con un poco sensato "vedendo che la Luna s'invecchia» ${ }^{\mathrm{I}}$.

Un'altra caratteristica che contraddistingue la versione dei due medici di Pralboino è, come ho già anticipato, l'utilizzo del dialetto veneziano, che, a seguito delle conquiste territoriali della Serenissima, si era propagato fino agli estremi confini occidentali segnati dal corso del fiume Adda (che ancora oggi separa la provincia di Bergamo da quella di Milano) ${ }^{15}$. Nei versi iniziali del prologo, per esempio, un termine dialettale è il verbo «impizza», usato al posto del più consueto «accendi» ${ }^{16}$; due altri esempi sono «nezza» (per «nipote», v. 47) e «scagno» (per «scanno» o «sgabello», v. 633$)^{17}$.

Altre volte, come abbiamo già visto, i riferimenti al mondo dei Rositini consistono nella sostituzione dei termini attici con i corrispondenti veneziani. Ai v. II8 e I250, il greco «obolo» è reso con «bagattino», che, proprio come il suo omologo attico, indicava una moneta piccola (il dodicesimo del soldo); il bagattino più famoso era proprio quello di Venezia, che era stato coniato dal $\mathrm{I}_{442}$ per le necessità di commercio minuto della terraferma (e che valeva un sesto del grosso). Allo stesso modo, al v. I235 il «triobolo» è reso con "quattrino», mentre al v. IO4I gli «stateri» diventano «ducati» ${ }^{18}$.

I4. Come al solito, Divus aveva tradotto letteralmente («videns agentem Lunam vigesimas»), lasciandoci il dubbio che non avesse colto pienamente il senso dell'espressione. I punti di contatto tra i Rositini e Divus sono talmente numerosi (e soprattutto, nella maggior parte dei casi, talmente evidenti) che non vale la pena dedicarvi molta attenzione.

I5. Brescia divenne veneziana nel I426 e Bergamo nel I428. Com’è noto, Venezia rischiò di perdere i possedimenti della Terraferma a causa della guerra mossa dagli eserciti del Papa e dei sovrani francesi, tedeschi e spagnoli (che avevano formato la Lega di Cambrai culminata nella sfortunata (per i Veneziani, che ne uscirono sconfitti) battaglia di Agnadello (I4 maggio I509); tuttavia nel I5ı6 la Serenissima riprese di nuovo il pieno possesso della Terraferma, che mantenne fino al trattato di Campoformio.

I6. Cfr. G. Boerio, Dizionario del dialetto veneziano, Venezia, Andrea Santini e figlio, I829, s.v. impizzar.

17. Cfr. Boerio, op. cit., alle voci nezza («la figlia di fratello o di sorella; ovv. La figliuola del figlio o della figlia rispetto all'avo ed all'ava») e scagno («arnese di legname sopra il quale si siede»). O. Pianigiani, Vocabolario etimologico della lingua italiana, Roma, Albrighi \& Segati, 1907, s.v. nezza, fa derivare il termine dal sostantivo neptis (testimoniato nella tarda latinità) attraverso la mediazione del provenzale netza, lo collega al francese nièce e lo definisce «una voce antica restata ai Veneziani e in qualche altro dialetto italiano per Nipote (femminile)"; anche se le etimologie del Pianigiani non sono sempre fondate, sia il Battaglia che il dizionario di Battisti-Alessio (C. Battisti e G. Alessio, Dizionario etimologico italiano, Firenze, Barbera, 1968) confermano il rapporto tra il termine tardo-latino e le sue derivazioni romanze. Anche la schiavina (cfr. supra, n. I2) è un termine dialettale: Boerio, op. cit., la definisce "Coperta da letto di lana ruvida e ben grossa».

I8. Mme Dacier (e pure Van Daele) traduce «obolo» con «obole», ma parafrasa sia la battuta del triobolo («Ho, oui par Jupiter, \& je me soûmets à payer l'amende si je ne jure») sia quella degli stateri («C'est un chose digne de toutes les couronnes \& de toutes les recompenses, que d'entreprendre les causes les plus mechantes \& de les gagner»), che Van Daele traduce con maggiore aderenza all’originale greco ("Oui, par Zeus, et j’ajouterais bien, pour pouvoir jurer, trois oboles» e "Et cet art-là vaut plus de dix mille statères, de prendre parti pour les raisons les plus faibles et, malgré cela, de l'emporter»). 
Ma il ricorso al dialetto risulta molto efficace soprattutto in un contesto ben preciso. La scena più famosa della commedia, l'agone tra il discorso giusto e il discorso ingiusto («Parola giusta» e «Parola ingiusta» per i fratelli Rositini, "La justice» e "L'injustice» per Mme Dacier), si conclude con la sconfitta del primo, costretto ad ammettere che ad Atene dominano i «larges-culs», secondo la resa di Van Daele, un insulto indicava coloro che avevano subìto quello che Kenneth Dover definisce perifrasticamente "enlargement of the anus by habitual subjection to anal coitus» ${ }^{19}$. Una signora come Mme Dacier non poteva tradurre letteralmente un termine

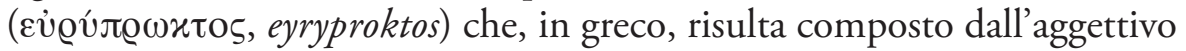

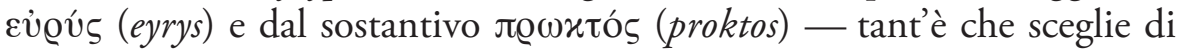
renderlo con il meno compromettente «infames». Più brillante (ma anche molto aderente al disinvolto humour artistofanesco) è la scelta dei Rositini, che, appoggiandosi sul significato osceno di «busòn» (un insulto che viene usato ancora ai nostri giorni), traducono l'espressione con «i larghi busi» ${ }^{20}$.

Sono proprio i contesti osceni quelli nei quali il dialetto conferisce alla traduzione una forza espressiva particolarmente efficace. Nelle Nuvole, casi simili sono abbastanza infrequenti, perché la trama della commedia offre raramente il pretesto a battute di stampo sessuale; in altre commedie, come per esempio la Lisistrata, gli esempi sono molto più numerosi. Quando, verso la fine della commedia, la protagonista comunica ai rappresentanti di Atene e Sparta le condizioni per porre fine allo sciopero del sesso, la personificazione della Tregua (una comparsa interpretata o da una donna o da un uomo vestito da donna) viene invitata a prendere l'ambasciatore spartano per mano - o, precisa Lisistrata, nel caso che l'ambasciatore non volesse dare alla Tregua la mano, di prenderlo per un'altra parte

19. Cfr. Aristophanes, Clouds, edited with introduction and commentary by K. J. Dover, Clarendon Press, Oxford 1968, v. I084; sul termine cfr. J. Henderson, The Maculate Muse. Obscene Language in Attic Comedy,

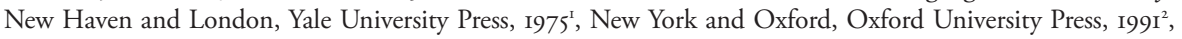
p. 2 IO.

20. La validità della resa dei Rositini è stata opportunamente sottolineata da Riccardo Quaglia, che, nel suo articolo dedicato alla storia delle traduzioni italiane delle commedie di Aristofane ( $S$ Su alcune traduzioni italiane di Aristofane: azzeccagarbugliando tra i secc. XVI e XIX", Maia, n. 57, 2006, pp. 349-357), scrive che «rendere gli દủ le sonorità venexiane di "larghi busi", adeguatamente ripetuto a mo', appunto, di tormentone, può strappare un sorriso» (p. 353). Sul significato di "busòn» cfr. Boerio, op. cit. («detto a uomo per ingiuria, Bardassa; Bagascia, Bagascione; Zanzero; Bastone»), che subito dopo menziona anche il femminile "busòna» («detto per agg. ed ingiuria a Femmina, Zambracca; Zambraccaccia, Puttana»); in un comizio tenuto a Bologna nel 2011 per le elezioni amministrative, Beppe Grillo ha salutato scherzosamente la folla con la frase "At salut, buson», che è stata interpretata da buona parte della stampa come un insulto rivolto al politico omosessuale Nichi Vendola.

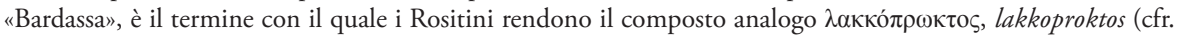
Henderson, op. cit., p. 2IO), vale a dire l'insulto che, dopo essere stato bastonato, Strepsiade lancia al figlio Fidippide al v. 1330 (e che Mme Dacier traduce coerentemente con «infame»). 
del corpo (v. III)). I Rositini traducono l'invito di Lisistrata con «menali il zuco», dove «zuco» sta per «ciocco» (cioè «bastone», secondo un processo metaforico facilmente comprensibile ${ }^{21}$. Poco più avanti, nella stessa scena, nel contemplare il posteriore della Tregua all'ambasciatore spartano scappa un giudizio molto elogiativo (v. II48), che Van Daele traduce con "Mais que cette croupe est ineffablement belle!". I Rositini non capiscono il senso di öфatov (aphaton, «non è possibile dire»), ma il resto sì, perché traducono l'esclamazione dello spartano con «il tomaso è inesplicabile e bello» - dove «tomaso» («Tommaso»), come accade con altri nomi propri maschili («toni» e «martin»), viene usato per indicare il culo ${ }^{22}$. Del resto, sempre nella Lisistrata troviamo anche il caso di un nome femminile usato per indicare il pudendum muliebre. Al v. 824, durante una delle tante schermaglie fra il semicoro maschile e quello femminile, gli uomini dicono alle donne che, se cercheranno di prenderli a calci, mostreranno la "filippa»: in questo caso il termine usato dai Rositini non è tanto dialettale quanto italiano, perché un uso analogo di «filippa» si trova anche in ambito linguistico toscano, come risulta da un canto carnascialesco di Alessandro di Rinaldo Bracci che celebra le virtù della trippa ${ }^{23}$.

Ma complessivamente non si tratta di soluzioni molto frequenti. Nella maggior parte dei casi i Rositini o si servono di termini propri o, più spesso, di eufemismi, necessari per attenuare la volgarità a volte eccessiva del testo di Aristofane. Sempre nel prologo, al v. 9, l'azione compiuta da Fidippide avvolto nelle cinque coperte di pelliccia è tradotta con «poltronegia» (dal verbo "poltroneggiare», cioè "giacere ozioso nel letto», come

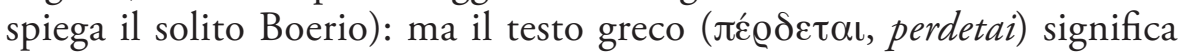
qualcosa di ben diverso (Van Daele: "pète»), come aveva capito perfino Andreas Divus, il quale aveva correttamente tradotto con pedit. Ci vorranno secoli prima che le flatulenze cessassero di suscitare scalpore in chi leggeva un testo letterario: prima di allora, prima che Claude-François Mercier de Compiègne scrivesse alla fine dell'Ottocento un celebre Éloge

2I. Secondo Boerio, op. cit., il termine veneziano è "zoco» ("coll'o stretto e la z aspra»). Poiché Boerio era nato a Lendinara, un paese vicino a Rovigo, ed era vissuto a Venezia, il suo dizionario riflette soprattutto varianti del dialetto di pianura; sulle montagne, esistevano (ed esistono) forme leggermente diverse, come per esempio proprio la variante "zuco" attestata nella lingua ladina. La traduzione francese di Van Daele (Aristophane, Comédies, Tome III : Les Oiseaux, Lysistrata, Paris, Les Belles Lettres, I928) è "conduis-le par le membre».

22. Cfr. Boerio, op. cit., s.v. tomào («voce ant. che voleva dire Tommaso, nome proprio di uomo - Detta ora per ischerzo, significa Culo, come Toni e Martin»).

23. Canti carnascialeschi I.553: «Dica pur chi vuol; la trippa/ sempre è 'l cibo il più pregiato, / tira a tutti la filippa,/ e solletica il palato»). Cfr. Battaglia, op. cit. ("Gerg. Ant. Vulva»). La traduzione di Van Daele («Tu feras

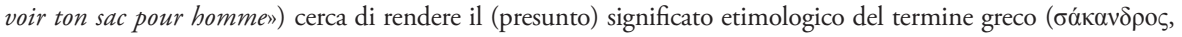

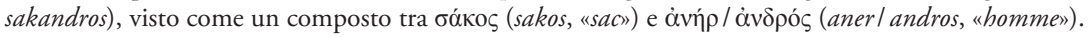


$d u$ pet, la tendenza era ricorrere all'eufemismo, come risulta dallo scontato «ronfle» usato da Mme Dacier.

Dopo queste due incursioni nel campo della letteratura, i fratelli Rositini preferirono coniugare la loro passione per il greco con i loro interessi professionali: dieci anni dopo Aristofane e Diogene Laerzio, nel I555, sempre a Venezia ma presso un altro editore («apud Balthasserem Constantinum, ad signum Divi Georgii»), pubblicarono - questa volta in traduzione latina - le Sententiae omnes ac verba, quae in divini Hippocratis aphorismis continentur, iam recens et accurate in novum ordinem alphabeticumque digesta, ut quaevis in ipsis commemorata nunc facilius et celerius quam antea inveniri queant, ut ita medicinae studiosis maiori nimirum, quàm prius, usui futura sint ${ }^{24}$; l'anno seguente, il 1556, il solo Pietro pubblicò il Trattato di mal francese, sempre a Venezia, per l'editore Lodovico Avanzi "alla libreria dall'Alboro»" ${ }^{25}$, nel 1558 sempre Pietro Rositini curò a Venezia, presso gli editori Vincenzo Valgrisio e Baldassarre Costantino (che nel frattempo avevano unito le loro tipografie), la pubblicazione della traduzione italiana del celebre trattato di chirurgia (Prattica utilissima et necessaria di cirugia) scritto nel I5I4 in latino dal medico rapallese Giovanni di Vico.

Per quel che ne sappiamo, non tornarono più a occuparsi del loro amato Aristofane. Ma nemmeno lo fecero altri intellettuali italiani: eliminando il poeta comico dal canone degli autori greci che si potevano leggere con profitto, la Controriforma cancellò di fatto Aristofane dal panorama culturale italiano fino alla metà del Novecento ${ }^{26}$, quando il conte Coriolano Malingri di Bagnolo, senatore del Regno di Sardegna, pubblicò la seconda traduzione integrale (in versi!) dell'opera omnia del poeta greco, stampata dall'editore Marzorati nel I850, cui fece seguito appena tre anni dopo, nel

24. La traduzione è dedicata a «Francesco Venerio», vale a dire Francesco Venier, che fu Doge di Venezia («serenissimus, illustrissimusque Venetiarum princeps») per soli due anni - e proprio dal I554 al I556.

25. Probabilmente Bartolomeo era morto durante la preparazione del volume ippocratico, perché nel frontespizio figurano entrambi i nomi («auctoribus et interpretibus fidissimis, Bartholomaeo et Petro Rostiniis, doctoribus physicis»), ma la lettera dedicatoria al Venier è scritta in prima persona ed è firmata dal solo «Petrus Rostinius».

26. Cfr. le parole di Carlo Prato in Aristofane, Le donne alle Tesmoforie, a cura di C. Prato, traduzione di D. Del Corno, Fondazione Lorenzo Valla, Milano, Mondadori, 20oI, p. xxxıII: «In seguito alla Controriforma (I545-63), l'insegnamento e lo studio del greco nel nostro paese furono ostacolati o duramente limitati, a tutto vantaggio del latino, assunto come lingua ufficiale non solo per la predicazione della dottrina cristiana, ma anche per la formazione della classe dirigente e dell'uomo colto. Gli autori greci ammessi furono, prima d'ogni altro, i Padri della Chiesa e, fra i pagani, i prosatori, in particolare Platone, Demostene, Tucidide, ecc.; Aristofane venne, si può dire, bandito dalla scuola per la sua "immoralità", come attestano le "circolari" inviate dai Gesuiti preposti alla direzione degli istituti secondari e delle Università. Questa situazione dura, tranne qualche eccezionale circostanza, propiziata da insigni umanisti o isolati cultori della classicità, per circa tre secoli, durante i quali il paese in cui era apparsa l'editio princeps dell'opera di Aristofane e nelle cui biblioteche giacevano numerosi i codici migliori, fu tagliato fuori da ogni iniziativa libraria, pur fiorente nel resto d'Europa, anche di quella in regime cattolico.» 
I853, la terza, ancora in prosa, dovuta a Domenico Capellina, professore di letteratura italiana all'Università di Torino ${ }^{27}$.

Fino ad allora, prima di essere coperte dall'oblio provocato da queste e da altre - più fortunate e, nel complesso, riuscite - versioni, le traduzioni naïves dei fratelli Bartolomeo e Pietro Rositini, fatte per compiacere un nobile amante delle letterature classiche, furono le prime che fecero risuonare nella lingua italiana la voce del «facetissimo" poeta. Aver potuto far risuonare di nuovo (benché solo sulla carta) questa voce è il giusto riconoscimento per un tentativo che, per quanto imperfetto, fa comunque onore a coloro che ci hanno provato.

\section{Bibliografia}

Aristofane, Le comedie del facetissimo Aristofane, tradutte di Greco in lingua commune d'Italia, per Bartolomeo \& Pietro Rositini de Prat' Alboino, Venezia, Vicenzo Vaugris, I545.

-, Le donne alle Tesmoforie, a cura di Carlo Prato, traduzione di Dario Del Corno, Fondazione Lorenzo Valla, Milano, Mondadori, 2001.

Aristophane, Aristophanis, comicorum principis, Comoediae undecim, ̀̀ Graeco in Latinum, ad verbum translatae, Andrea Divo Iustinopolitano interprete, trad. Andreas Divus, Venetijs: apud d. Iacob a Burgofrancho Papiensem, 1538.

—, Le Plutus et les Nuées d'Aristophane, comédies grecques, trad. Mme Dacier, Parigi, Denys Thierry et Claude Barbin, I684.

-, Comédies, Tome I: Les Acharniens, Les Cavaliers, Les Nuées, texte établi par Victor Coulon et trad. française de Hilaire Van Daele, Paris, Les Belles-Lettres, 1923, rééd. 1934, 1948...; Tome III : Les Oiseaux, Lysistrata, trad. française de Hilaire Van Daele, Paris, Les Belles Lettres, 1928.

Aristophanes, Clouds, edited with introduction and commentary by Kenneth James Dover, Clarendon Press, Oxford 1968.

Battaglia Salvatore, Grande dizionario della lingua italiana, Torino, UTET, i96r.

Battisti Carlo e Alessio Giovanni, Dizionario etimologico italiano, Firenze, Barbera, 1968 ( ${ }^{\text {re }}$ éd. 1940).

27. Su queste traduzioni cfr. Quaglia, op. cit.; sulle traduzioni successive cfr. M. L. Chirico, «Aristofane nel XIX secolo in Italia. Domenico Comparetti e Augusto Franchetti», I quaderni di Atene e Roma, 3, 20I2, pp. 3II334, ripreso nel saggio "Translations of Aristophanes in Italy in the I9th century», in corso di stampa. 
Beta Simone, «La prima traduzione latina della Lisistrata. Luci e ombre della versione di Andrea Divo", Quaderni Urbinati di Cultura Classica, n.s. IOO, n. I, 2012 (vol. I29 della serie continua), pp. 95-II4.

Boerio Giuseppe, Dizionario del dialetto veneziano, Venezia, Andrea Santini e figlio, I829.

Chirico Maria Luisa, "Aristofane nel xix secolo in Italia. Domenico Comparetti e Augusto Franchetti», I quaderni di Atene e Roma, 3, 2012, pp. 3II-334.

Colvin Stephen, Dialect in Aristophanes and the Politics of Language in Ancient Greek Literature, Oxford, Clarendon Press, I999.

Farnham Fern, Madame Dacier: Scholar and Humanist, Monterey, Angel Press, 1976.

Henderson Jeffrey, The Maculate Muse. Obscene Language in Attic Come$d y$, New Haven and London, Yale University Press, 1975'1 New York and Oxford, Oxford University Press, I991 ${ }^{2}$.

Malcovati Enrica, Madame Dacier: una gentildonna filologa del gran secolo, Firenze, Sansoni, 1952.

Mazon Paul, Mme Dacier et les traductions d'Homère en France, Oxford, Clarendon Press, 1936.

Nassichuk John, 'Strepsiades' Latin Voice: Two Renaissance Translations of Aristophanes' Clouds, in corso di stampa.

Pagani Giuseppe, "Mario Nizzoli e il suo lessico", in Rendiconti della Reale Accademia dei Lincei, s. 5, II, I893, pp. 554-575; "Le polemiche letterarie di Mario Nizzoli», ibid., pp. 630-66o; "Mario Nizzoli umanista e filosofo del sec. XVI», ibid., pp. 716-74I; "Operosità letteraria di Mario Nizzoli», ibid., pp. 819-826; "Gli ultimi anni di vita di Mario Nizzoli», ibid., pp. 897-922.

Palumbo Margherita, «Nizzòli (Nizolio), Mario», in Dizionario biografico degli italiani, vol. 78, pp. 620-623.

Pianigiani Ottorino, Vocabolario etimologico della lingua italiana, Roma, Albrighi \& Segati, I907.

QuAglia Riccardo, «Su alcune traduzioni italiane di Aristofane: azzeccagarbugliando tra i secc. XVI e XIX», Maia, n. 57, 2006, pp. 349-357.

Santangelo Giovanni Saverio, Madame Dacier, una filologa nella crisi: I672-I720, Roma, Bulzoni, I984.

Tesi Riccardo, Aristotele in italiano. I grecismi nelle traduzioni rinascimentali della 'Poetica', Firenze, Accademia della Crusca, 1997.

Tiraboschi Girolamo, Biblioteca modenese o Notizie della vita e delle opere degli scrittori natii degli stati del serenissimo signor Duca di Modena, Modena, Società tipografica, 1783. 